\title{
Incidence des traitements insecticides sur la faune aranéologique d'un champ de blé à l'épiaison.
}

\author{
C Cocquempot, JP Chambon, P Reynaud, L Fischer \\ INRA, Station de Zoologie, Laboratoire de Faunistique, 78026 Versailles Cedex, France
}

(Reçu le 8 mars 1989; accepté le 14 mars 1991)

\begin{abstract}
Résumé - Ce travail fait le point sur l'incidence de 3 matières actives insecticides (deltaméthrine, diméthoate et phosalone) sur les araignées des champs de blé dans le cas de leur utilisation en traitement de végétation contre le puceron de épis (Sitobion avenae) à l'épiaison. L'inventaire des araignées comporte 110 espèces. L'action des produits est étudiée par comparaison des effectifs, des parcelles traitées entr'elles et avec le témoin, par une analyse de variance pour tous les cas où ils sont suffisants. Les résultats des analyses sont présentés sous la forme de tableaux et commentés dans le texte, pour les espèces les plus abondamment capturées : Pardosa prativaga, Erigone atra, Oedothorax apicatus, Theridion bimaculatum et Lepthyphantes tenuis ainsi que pour l'ensemble des Erigonidae. Globalement il ressort de cette étude que les araignées sont relativement peu affectées par les produits étudiés, l'effet étant souvent de courte durée. Par ailleurs, dans de nombreux cas, il semble que les matières actives étudiées ne tuent pas ces arthropodes mais perturbent leur activité durant un laps de temps variable selon les produits et les espèces.
\end{abstract}

araignée / prédateur polyphage / activité / blé / traitement insecticide

Summary - Incidence of insecticide treatment on araneological fauna in wheat field at flowering stage. The aim of this study was to evaluate the incidence on spiders in wheat fields of 3 active insecticide ingredients (deltamethrin, dimethoate and phosalone) used against the English grain aphid (Sitobion avenae) at flowering stage. One hundred and ten species have been listed. Incidence has been studied by comparison of numbers of captures between different treated and untreated plots. The results have been statistically analysed using variance analysis only when the numbers were sufficient. The results of the analysis have been presented in the tables and commented on in the text for the main species caught: Pardosa prativaga, Erigone atra, Oedothorax apicatus, Theridion bimaculatum, Lepthyphantes tenuis and the Erigonidae. Spiders are slightly affected by insecticides and the effect is often brief. However, although the active ingredients studied do not kill these arthropods, they seem to disturb their activity over a variable period depending upon the insecticide and the species.

spiders / polyphagous predators / activity / wheat / insecticide treatment

\section{INTRODUCTION}

Les travaux européens (Vickerman et Sunderland, 1977; De Clercq et Pietraszko, 1983; Basedow et al, 1985; Powell et al, 1985) montrent l'existence d'un effet néfaste sur les araignées, plus ou moins durable et différent selon les matières actives insecticides. En France, dans une étude précédente, Fischer et Chambon (1987a) ont montré l'incidence des traitements insecticides sur l'entomofaune et mentionnent de façon globale cette action sur la faune aranéologique.

Les travaux rapportés dans cet article concernent l'action comparée de $\mathbf{3}$ matières ac- tives utilisées sur céréales en France (deltaméthrine, phosalone, diméthoate) en traitement contre le puceron des épis (Sitobion avenae (F)) au début de la floraison.

Les observations portent sur les araignées qui représentent une part importante des prédateurs polyphages présents dans une biocénose céréalière (Cocquempot et Chambon, 1989b). Cette étude implique la connaissance qualitative et quantitative de la faune présente au moment de l'intervention et le suivi régulier de l'évolution des effectifs de captures, obtenus par diverses techniques de recensement dans les différentes situations expérimentales. 
Le bilan de cette recherche montre l'action des 3 matières actives sur les araignées (adultes). II apporte des éléments d'appréciation pour l'utilisation rationnelle des insecticides et contribue ainsi à l'élaboration d'une stratégie de lutte raisonnée en céréaliculture.

\section{MATÉRIELS ET MÉTHODES}

L'expérimentation a été menée, en collaboration avec la société Procida/Roussel-Uclaf, en 1983, 1984 et 1985, dans un champ de blé de 18,5 ha situé à SaintCyr-en-Arthies (Val-d'Oise) (fig 1).

Les traitements insecticides ont été réalisés les 20 juin 1983, 19 juin 1984 et 20 juin 1985 (tableau I). Ces dates correspondent au stade phénologique compris entre le début et la mi-floraison du blé. Les conditions d'application des produits ont été les suivantes :

- rampe de pulvérisation de 18 mètres équipée de jets pinceaux se croisant au niveau des épis;

- la pression de pulvérisation était de 2 bars;

- les quantités d'eau étaient de 300 t/ha en 1983 et 200 l/ha en 1984 et 1985;

- les doses d'application des matières actives étaient de :

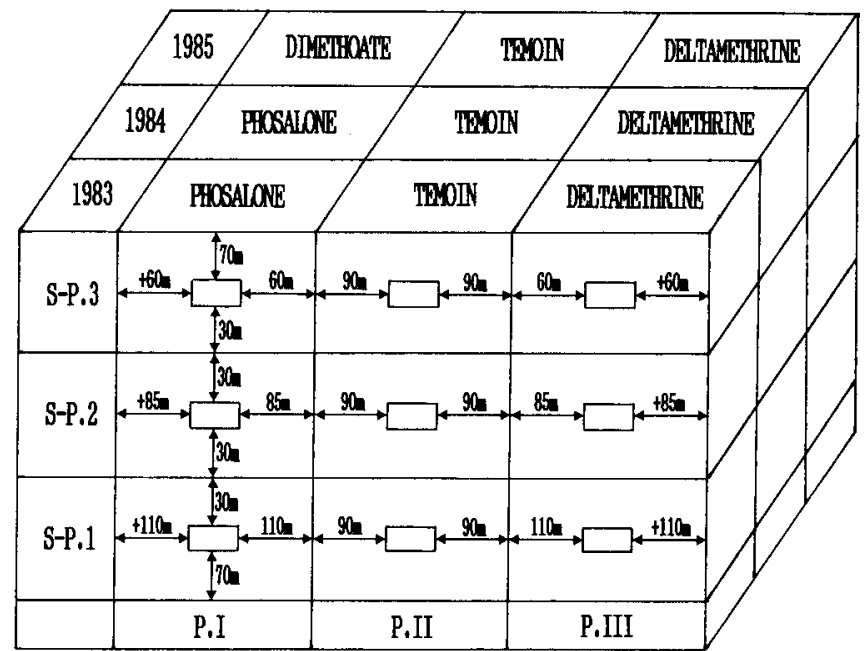

Fig 1. Représentation schématique de l'aire expérimentale en fonction de l'année. $P$ = Parcelle; $S-P=$ Sous-parcelle; $=$ Emplacement du poste de piégeage.

$-600 \mathrm{~g} / \mathrm{ha}$ pour la phosalone;

$-6,25 \mathrm{~g} / \mathrm{ha}$ pour la deltaméthrine;

$-400 \mathrm{~g} / \mathrm{ha}$ pour le diméthoate.

Tableau I. Calendrier des prélèvements (pots-pièges, plateaux colorés, D-VAC). ${ }^{*}=$ Relevés quotidiens pour les pots pièges et les plateaux colorés; $\mathrm{TT}=$ Traitement.

\begin{tabular}{|c|c|c|c|c|c|}
\hline & \multicolumn{3}{|c|}{ Relevés pots-pièges et plateaux colorés } & \multicolumn{2}{|c|}{ Aspirations au D-VAC } \\
\hline & 1983 & 1984 & 1985 & 1984 & 1985 \\
\hline Période 1 & $\begin{array}{l}3 \text { relevés du } \\
6 \text { juin au } 13 \text { juin }\end{array}$ & $\begin{array}{c}3 \text { rélevés du } \\
4 \text { juin au } 12 \text { juin }\end{array}$ & $\begin{array}{c}3 \text { relevés du } \\
5 \text { juin au } 13 \text { juin }\end{array}$ & & \\
\hline Période 2 & $\begin{array}{l}2 \text { relevés du } \\
13 \text { juin au } 20 \text { juin } \\
\text { avant } \pi\end{array}$ & $\begin{array}{l}4 \text { relevés du } \\
12 \text { juin au } 19 \text { juin } \\
\text { avant TT }\end{array}$ & $\begin{array}{c}5 \text { relevés du } \\
13 \text { juin au } 20 \text { juin } \\
\text { avant TT }\end{array}$ & $\begin{array}{l}2 \text { relevés } \\
15 \text { et } 19 \text { juin } \\
\text { avant TT }\end{array}$ & $\begin{array}{l}2 \text { relevés } \\
14 \text { et } 20 \text { juin } \\
\text { avant TT }\end{array}$ \\
\hline Traitement & 20 juin & 19 juin & 20 juin & 19 juin & 20 juin \\
\hline Périodes $3^{*}$ & $\begin{array}{l}7 \text { relevés du } \\
20 \text { juin après } \\
T T \text { au } 27 \text { juin }\end{array}$ & $\begin{array}{l}7 \text { relevés du } \\
19 \text { après } \\
T T \text { au } 26 \text { juin }\end{array}$ & $\begin{array}{l}7 \text { relevés du } \\
20 \text { juin après } \\
\text { au } 27 \text { juin }\end{array}$ & $\begin{array}{l}2 \text { relevés } \\
20 \text { et } 22 \text { juin }\end{array}$ & $\begin{array}{l}4 \text { relevés } \\
21,22,25 \\
\text { et } 26 \text { juin }\end{array}$ \\
\hline Période $4^{*}$ & $\begin{array}{l}7 \text { relevés du } \\
27 \text { juin au } 4 \text { juill }\end{array}$ & $\begin{array}{c}7 \text { relevés du } \\
26 \text { juin au } 3 \text { juill }\end{array}$ & $\begin{array}{c}6 \text { relevés du } \\
27 \text { juin au } 3 \text { juill }\end{array}$ & $\begin{array}{c}2 \text { relevés } \\
29 \text { juin au } 2 \text { juill }\end{array}$ & $\begin{array}{c}2 \text { relevés } \\
28 \text { juin au } 2 \text { juill }\end{array}$ \\
\hline Période 5 & $\begin{array}{l}3 \text { relevés du } \\
4 \text { juill au } 12 \text { juill }\end{array}$ & $\begin{array}{l}3 \text { relevés du } \\
3 \text { juill au } 12 \text { juill }\end{array}$ & $\begin{array}{c}3 \text { relevés du } \\
3 \text { juill au } 11 \text { juill }\end{array}$ & $\begin{array}{l}2 \text { relevés } \\
5 \text { et } 9 \text { juill }\end{array}$ & $\begin{array}{l}2 \text { relevés } \\
5 \text { et } 9 \text { juill }\end{array}$ \\
\hline Période 6 & & $\begin{array}{c}2 \text { relevés du } \\
12 \text { juill au } 20 \text { juill }\end{array}$ & $\begin{array}{c}3 \text { relevés du } \\
11 \text { juill au } 23 \text { juill }\end{array}$ & $\begin{array}{c}3 \text { relevés } \\
13,16 \text { et } \\
20 \text { juill }\end{array}$ & $\begin{array}{c}4 \text { relevés } \\
12,15,19 \\
\text { et } 23 \text { juill }\end{array}$ \\
\hline Période 7 & & $\begin{array}{c}2 \text { relevés du } \\
20 \text { juill au } 31 \text { juill }\end{array}$ & & $\begin{array}{l}2 \text { relevés } \\
26 \text { et } 31 \text { juill }\end{array}$ & \\
\hline
\end{tabular}


Des observations complémentaires à l'expérimentation (Fischer, 1987) ont permis de montrer que, lors d'une pulvérisation à 2 bars de pression, la quantité de produit atteignant les différentes strates de la végétation diminue de manière très sensible, de l'épi vers les premières feuilles, et devient très faible au niveau du sol.

Les prélèvements ont été effectués à l'aide de 18 pots pièges et de 36 plateaux colorés répartis en 3 postes de piégeage (S-P 1,2,3) distribués dans chacune des trois parcelles (P I, II, III) de 6 ha environ (fig 1).

Les postes de piégeage étaient composés de 2 pots pièges de $11 \mathrm{~cm}$ de diamètre, de 2 plateaux colorés maintenus au niveau supérieur de la végétation et 2 plateaux colorés posés sur le sol. Chaque piège était séparé des autres par au moins $5 \mathrm{~m}$ à l'intérieur d'un même poste. Le dispositif de piégeage était opérationnel en permanence, les pots et les plateaux étant remis en place immédiatement après chaque relevé.

En 1984 et 1985, des prélèvements par aspiration au D-VAC ont complété le dispositif. Les modalités de ces prélèvements étaient de 100 ponctions de 2-3 s chacune par parcelle en 1984 et de 33 ponctions par sous-parcelle (99 par parcelle) en 1985.

L'ensemble des informations de ce chapitre sont détaillées dans de précédents travaux (Fischer, 1987; Fischer et Chambon, 1987a, 1987b; Reynaud, 1989).

Le calendrier des prélèvements (pots pièges, plateaux colorés, D-VAC) est donné dans le tableau I.

L'analyse taxonomique du matériel récolté a été réalisée sur la base des ouvrages de systématique de Simon (1914-1932), Locket et Millidge (1951-1953), Wiehle (1956, 1960), Tongiorgi (1966), Locket et al (1974) et de Ledoux et Canard (1981).

L'analyse statistique a consisté à tester l'homogénéité des effectifs des parcelles traitées par rapport à la parcelle témoin. La méthode utilisée est l'analyse de variance par le test au $T$ de Fischer. Les valeurs testées ont été préalablement transformées en $L(x+1)$. Quand l'analyse de variance globale a révélé une différence significative, nous avons utilisé la ppds pour comparer les parcelles 2 à 2 (méthode ppds protégée) (Snedecor et Cochran, 1957).

Cette analyse des données n'est réalisable que si les effectifs sont suffisants. Cette contrainte nous a conduits à opérer le regroupement des prélèvements en 5-7 périodes de 7-11 j, selon les années (tableau I), le traitement intervenant entre les périodes 2 et 3.

Dans le cas de différences significatives constatées avant traitement, on peut conclure à une hétérogénéité de l'aire d'expérimentation et interpréter les résultats avec la plus grande prudence. Dans le cas où aucune différence significative n'a été constatée avant le traitement, si on note une différence après traitement, on peut conclure à un effet de la matière active sur l'espèce étudiée.

La différence des captures après/avant traitement, exprimée en pourcentage des captures avant traitement (toutes sous-parcelles et périodes confondues) a été calculée pour chaque parcelle et chaque année. Ces données permettent de comparer l'évolution des captures dans le temps entre le témoin et les parcelles traitées et d'affiner l'interprétation des résultats de l'analyse statistique.

\section{RÉSULTATS}

Liste systématique des 110 espèces d'araignées recensées au cours de l'étude.

\section{Dyctinidae}

Argenna subnigra (OP Cambridge)

\section{Gnaphosidae}

Drassodes lapidosus (Walckenaer)

Haplodrassus dalmatensis (L Koch)

Zelotes lutetianus (L Koch)

Zelotes pusillus (CL Koch)

Zelotes praeficus (L Koch)

\section{Clubionidae}

Clubiona reclusa OP Cambridge

Clubiona terrestris Westring

Clubiona similis L Koch

Clubiona compta CL Koch

Clubiona brevipes Blackwall

Clubiona subtilis L Koch

Agroeca brunnea (Blackwall)

\section{Zoridae}

Zora spinimana (Sundevall)

\section{Anyphaenidae}

Anyphaena accentuata (Walckenaer)

\section{Thomisidae}

Misumena vatia (Clerck)

Xysticus cristatus (Clerck)

Xysticus Kochi Thorell

Xysticus lanio CL Koch

Xysticus ulmi (Hahn)

Oxyptila simplex (OP Cambridge)

\section{Philodromidae}

Philodromus dispar Walckenaer

Philodromus aureolus (Clerck)

Tibellus oblongus (Walckenaer) 


\section{Salticidae}

Salticus zebraneus (CL Koch)

Heliophanus flavipes (Hahn)

Euophrys aequipes (OP Cambridge)

\section{Lycosidae}

Pardosa monticola (Clerck)

Pardosa palustris (Linnaeus)

Pardosa pullata (Clerck)

Pardosa prativaga (L Koch)

Pardosa lugubris (Walckenaer)

Trochosa ruricula (Degeer)

Trochosa terricola Thorell

Arctosa perita (Latreille)

Pirata piraticus (Clerck)

Pirata latitans (Blackwall)

Aulonia albimana (Walckenaer)

\section{Pisauridae}

Pisaura mirabilis (Clerck)

\section{Hahniidae \\ Hahnia sp \\ Mimetidae \\ Ero aphana (Walckenaer) \\ Ero furcata (Villers)}

\section{Theridiidae}

Episinus angulatus (Blackwall)

Episinus truncatus Latreille

Crustulina sticta (OP Cambridge)

Anelosimus vittatus $\mathrm{CL}$ Koch

Achaearanea lunata (Clerck)

Theridion sisyphium (Clerck)

Theridion varians Hahn

Theridion mystaceum L Koch

Theridion tinctum (Walckenaer)

Theridion bimaculatum (Linnaeus)

Theridion pallens Blackwall

Theridion suaveolens Simon

Enoplognatha ovata (Clerck)

Enoplognatha thoracica (Hahn)

Enoplognatha schaufussi (L Koch)

Robertus lividus (Blackwall)

Robertus neglectus (OP Cambridge)

\section{Tetragnathidae}

Tetragnatha extensa (Linnaeus)

Pachygnatha degeeri Sundevall

\section{Araneldae}

Araneus bituberculatus Walckenaer

Araneus gibbosus (Walckenaer)

Araneus adiantus Walckenaer

Araneus sturmi (Hahn)

Araneus cucurbitinus Clerck

Hypsosinga pygmaea (Sundevall)

Mangora acalypha (Walckenaer)

\section{Erigonidae}

Walckenaera antica (Wider)

Walckenaera melanocephala (OP Cambridge)

Walckenaera nudipalpis (Westring)

Walckenaera corniculans (OP Cambridge)

Walckenaera vigilax (Blackwall)

Entelecara flavipes (Blackwall)

Entelecara graeca (OP Cambrigde)

Trematocephalus cristatus (Wider)

Hypomma bituberculatum (Wider)

Maso gallica Simon

Pocadicnemis pumila (Blackwall)

Oedothorax fuscus (Blackwall)

Oedothorax retusus (Westring)

Oedothorax apicatus (Blackwall)

Pelecopsis parallela (Wider)

Tiso vagans (Blackwall)

Gongylidiellum vivum (OP Cambridge)

Gongylidiellum latebricola (OP Cambridge)

Congylidiellum calcariferum Simon

Micrargus herbigradus (Blackwall)

Micrargus subaequalis (Westring)

Diplocephalus latifrons (OP Cambridge)

Araeoncus humilis (Blackwall)

Panamomops sulcifrons (Wider)

Milleriana inerrans (OP Cambridge)

Erigone dentipalpis (Wider)

Erigone atra (Blackwall)

Erigone vagans Audouin

\section{Linyphiidae}

Porrhomma microphtalmum (OP Cambridge)

Agyneta subtilis (OP Cambridge) 
Meioneta rurestris (CL Koch)

Microneta viaria (Blackwall)

Hylyphantes nigritus (Simon)

Centromerus sp

Oreonetides abnormis (Blackwall)

Bathyphantes gracilis (Blackwall)

Diplostyla concolor (Wider)

Lepthyphantes obscurus (Blackwall)

Lepthyphantes ericaeus (Blackwall)

Lepthyphantes tenuis (Blackwall)

Prolinyphia emphana (Walckenaer)

Microlinyphia pusilla (Sundevall)

Avec 110 espèces, la liste de Saint-Cyr-enArthies est légèrement plus longue que celle établie dans le Gâtinais (104) (Cocquempot et Chambon, 1989a). Les deux listes présentent néanmoins de grandes similitudes. En particulier : 57 espèces sont communes aux 2 régions et représentent dans les 2 cas plus de $90 \%$ des effectifs d'adultes capturés et la plupart des espèces dominantes sont les mêmes (Pardosa prativaga, Erigone atra, E dentipalpis, Oedothorax apicatus, Bathyphantes gracilis, Lepthyphantes tenuis, Theridion bimaculatumL. Les légères différences observées ont vraisemblablement pour origine d'une part, la zone étudiée, la pratique de la monoculture du blé qui a pu favoriser la faune aranéologique et la proximité d'un environnement boisé sur 3 côtés qui explique l'abondance des Lycosidae et la présence d'un grand nombre d'espèces à très faibles effectifs et d'autre part, les techniques de prélèvements mises en œuvre. En effet l'emploi du D-VAC a permis d'appréhender une partie de la faune vivant dans la strate supérieure de la végétation et explique le recensement de nombreux Theridiidae et Araneidae.

\section{Incidence des traitements sur les espèces d'araignées les plus abondamment capturées}

Seules sont, présentées dans les tableaux suivants les données concernant les adultes des espèces pour lesquelles des différences significatives ont été constatées durant au moins une période.

\section{Les Lycosidae (Pardosa prativaga) (tableau II)}

Les analyses faites sur des effectifs relativement faibles, obtenus en plateaux colorés, n'ont pas fait apparaître de différence significative impu- table à l'une ou l'autre des matières actives, quelle que soit l'année. Par contre Pardosa prativaga, la plus abondante des 11 espèces de Lycosidae recensées avec $91 \%$ des effectifs de cette famille (adultes), particulièrement bien interceptée par les pots-pièges, montre une certaine sensibilité aux insecticides.

Alors qu'aucune différence significative n'a été constatée en 1985, les chiffres en font apparaître, pour la période 3 de 1983 et 1984, entre le témoin, la deltaméthrine et la phosalone.

Les effectifs notés dans les parcelles traitées à la deltaméthrine sont, pour les 2 années, très significativement inférieurs à ceux du témoin. En 1983, la phosalone montre un effet singificatif défavorable. On note, par contre, une différence très significative en faveur de la parcelle traitée à la phosalone en 1984. Cette différence se retrouve au niveau des pourcentages d'évolution des captures après et avant traitement (+ $18,3 \%$ contre - $11 \%$ dans le témoin).

Dans l'état actuel de nos connaissances et en fonction des techniques de prélèvements utilisées, il ne nous est pas possible de préciser si l'évolution des captures constatée dans la parcelle phosalone est due aux effectifs naturels plus élevés (ces différences n'étant pas toutefois significatives avant le traitement) ou à une activité exacerbée par le traitement.

\section{Les Erigonidae (tableau III), Erigone atra (tableau IV), Oedothorax apicatus (tableau V)}

Les Erigonidae (tableau III) vivent au niveau du sol. La technique du pot piège est bien adaptée à leur capture et permet d'obtenir des effectifs suffisants pour être analysés.

Des différences sont notées en 1983 et 1985 entre le témoin et la deltaméthrine. Cette matière active marque de façon très significative les populations d'Erigonidae durant 2-3 semaines après traitement.

Le diméthoate ne semble pas avoir affecté cette famille, la seule différence significative avec le témoin est en faveur de la parcelle traitée lors de la sixième période en 1985.

L'étude des pourcentages d'évolution des captures corrobore les tests statistiques de 1983. En 1985, la progression des effectifs d'Erigonidae est très forte dans le témoin $(+988 \%)$, relativement faible dans la parcelle deltaméthrine $(+117 \%)$. Dans la parcelle diméthoate, cette progression (+ $591 \%$ ) est nettement inférieure à celle du témoin, malgré la dif- 
Tableau II. Effectifs, évolution pré/post traitement des captures et analyses statistiques sur Pardosa prativaga en pots-pièges. \% ap/av TT = différence des captures après/avant traitement, exprimée en pourcentage des captures d'avant traitement.

\begin{tabular}{|c|c|c|c|c|c|c|c|c|c|c|c|c|}
\hline & \multicolumn{3}{|c|}{$\begin{array}{c}\text { Témoin } \\
\text { Sous-parcelles }\end{array}$} & \multicolumn{3}{|c|}{$\begin{array}{l}\text { Deltaméthrine } \\
\text { Sous-parcelles }\end{array}$} & \multicolumn{3}{|c|}{$\begin{array}{c}\text { Phosalone } \\
\text { Sous-parcelles }\end{array}$} & \multicolumn{3}{|c|}{$\begin{array}{l}\text { Tests entre } \\
\text { les parcelles }\end{array}$} \\
\hline & 1 & 2 & 3 & 1 & 2 & 3 & 1 & 2 & 3 & $D T$ & $P T$ & $D F$ \\
\hline \multicolumn{13}{|l|}{1983} \\
\hline $\begin{array}{l}\text { Période } 1 \\
\text { Période } 2\end{array}$ & $\begin{array}{l}12 \\
38\end{array}$ & $\begin{array}{r}13 \\
4\end{array}$ & $\begin{array}{l}21 \\
31\end{array}$ & $\begin{array}{r}3 \\
10\end{array}$ & $\begin{array}{r}7 \\
17\end{array}$ & $\begin{array}{r}17 \\
6\end{array}$ & $\begin{array}{r}9 \\
21\end{array}$ & $\begin{array}{l}14 \\
39\end{array}$ & $\begin{array}{r}5 \\
23\end{array}$ & $\begin{array}{l}\text { NS } \\
\text { NS }\end{array}$ & $\begin{array}{l}\text { NS } \\
\text { NS }\end{array}$ & $\begin{array}{l}\text { NS } \\
\text { NS }\end{array}$ \\
\hline \multicolumn{13}{|l|}{ Traitement } \\
\hline $\begin{array}{l}\text { Période } 3 \\
\text { Période } 4 \\
\text { Période } 5\end{array}$ & $\begin{array}{l}51 \\
23 \\
26\end{array}$ & $\begin{array}{r}36 \\
7 \\
8\end{array}$ & $\begin{array}{l}53 \\
24 \\
13\end{array}$ & $\begin{array}{l}8 \\
5 \\
0\end{array}$ & $\begin{array}{l}14 \\
15 \\
18\end{array}$ & $\begin{array}{r}7 \\
7 \\
13\end{array}$ & $\begin{array}{l}27 \\
27 \\
20\end{array}$ & $\begin{array}{r}33 \\
21 \\
8\end{array}$ & $\begin{array}{l}40 \\
20 \\
21\end{array}$ & $\begin{array}{l}++ \\
\text { NS } \\
\text { NS }\end{array}$ & $\begin{array}{l}+ \\
\text { NS } \\
\text { NS }\end{array}$ & $\begin{array}{l}\underline{H} \\
\text { NS } \\
\text { NS }\end{array}$ \\
\hline $\begin{array}{l}\% \text { ap/av TT } \\
1984\end{array}$ & & 102 & & & +45 & & & 95,5 & & & & \\
\hline $\begin{array}{l}\text { Période } 1 \\
\text { Période } 2\end{array}$ & $\begin{array}{l}16 \\
28\end{array}$ & $\begin{array}{l}15 \\
25\end{array}$ & $\begin{array}{l}28 \\
33\end{array}$ & $\begin{array}{r}4 \\
19\end{array}$ & $\begin{array}{r}9 \\
46\end{array}$ & $\begin{array}{l}18 \\
39\end{array}$ & $\begin{array}{l}29 \\
58\end{array}$ & $\begin{array}{l}27 \\
49\end{array}$ & $\begin{array}{l}26 \\
35\end{array}$ & $\begin{array}{l}\text { NS } \\
\text { NS }\end{array}$ & $\begin{array}{l}\text { NS } \\
\text { NS }\end{array}$ & $\begin{array}{l}\text { NS } \\
\text { NS }\end{array}$ \\
\hline \multicolumn{13}{|l|}{ Traitement } \\
\hline $\begin{array}{l}\text { Période } 3 \\
\text { Période } 4 \\
\text { Période } 5 \\
\text { Période } 6 \\
\text { Période } 7\end{array}$ & $\begin{array}{r}26 \\
26 \\
23 \\
3 \\
8\end{array}$ & $\begin{array}{r}14 \\
7 \\
1 \\
1 \\
1\end{array}$ & $\begin{array}{r}10 \\
3 \\
0 \\
5 \\
1\end{array}$ & $\begin{array}{l}6 \\
6 \\
4 \\
0 \\
3\end{array}$ & $\begin{array}{r}6 \\
11 \\
7 \\
5 \\
10\end{array}$ & $\begin{array}{r}11 \\
14 \\
20 \\
6 \\
16\end{array}$ & $\begin{array}{r}26 \\
29 \\
9 \\
0 \\
8\end{array}$ & $\begin{array}{r}45 \\
37 \\
11 \\
3 \\
8\end{array}$ & $\begin{array}{r}37 \\
17 \\
16 \\
3 \\
16\end{array}$ & $\begin{array}{l}++ \\
\text { NS } \\
\text { NS } \\
\text { NS } \\
\text { NS }\end{array}$ & $\begin{array}{l}\star \star \\
\text { NS } \\
\text { NS } \\
\text { NS } \\
\text { NS }\end{array}$ & $\begin{array}{l}\text { H } \\
\text { NS } \\
\text { NS } \\
\text { NS } \\
\text { NS }\end{array}$ \\
\hline$\%$ ap/av TT & \multicolumn{3}{|c|}{$-11 \%$} & \multicolumn{3}{|c|}{$-7,4 \%$} & \multicolumn{3}{|c|}{$+18,3 \%$} & & & \\
\hline
\end{tabular}

$\mathrm{T}=$ Témoin, $\mathrm{D}=$ Deltaméthrine, $\mathrm{P}:$ Phosalone, $\mathrm{R}=$ Diméthoate. $\mathrm{NS}=$ Non significatif; $+=$ Différence significative au seuil de $5 \%$ en faveur du témoin; ++ = Différence très significative au seuil de $1 \%$ en faveur du témoin; * = Différence significative au seuil de $5 \%$ en faveur de la parcelle traitée; ${ }^{* \star}=$ Différence très significative au seuil de $1 \%$ en faveur de la parcelle traitée; $\pm=$ Différence significative au seuil de $5 \%$ en faveur de la seconde matière active; \pm = Différence très significative au seuil de $1 \%$ en faveur de la seconde matière active; ${ }^{\circ}=$ Différence très significative au seuil de $1 \%$ en faveur de la première matière active.

férence significative supérieure constatée lors de la période 6, qui n'est probablement que la conséquence des effectifs initiaux plus élevés dans cette parcelle.

Cette évolution des effectifs laisse penser que l'action du diméthoate occasionne une simple réduction de l'activité des Erigonidae sur les 3 premières périodes après traitement, alors que celle de la deltaméthrine entraîne une forte réduction pouvant traduire une certaine mortalité.

Erigone atra (tableau IV), araignée agrophile vivant au niveau du sol, très abondante dans les milieux céréaliers du Bassin parisien (Cocquempot et Chambon, 1989a), présente des effectifs suffisants uniquement en pots pièges. Les populations d'E atra sont affectées par la deltaméthrine et la phosalone en 1983, ainsi que par la deltaméthrine et le diméthoate en 1985. Des différences significatives sont enregistrées au détriment des parcelles traitées durant des périodes variables. La deltaméthrine affecte les populations d'E atra pendant 2 (1985) à 3 (1983) périodes après traitement. La baisse significative des captures notée en période 5 (plus de 15 j après le traitement), dans les parcelles traitées à la phosalone en 1983, ne se retrouve pas au niveau des captures globales après traitement par rapport au témoin. Cette différence est difficilement imputable à l'action de la matière active dont la rémanence n'est que de 15-18 j et ne peut être vérifiée en raison de l'arrêt des prélèvements.

En 1985, le diméthoate montre les mêmes caractéristiques que la deltaméthrine. Les différences hautement significatives enregistrées avant traitement portent sur de très faibles effectifs et n'affectent pas les résultats notés après traitement. Les pourcentages calculés par parcelle montrent que l'évolution des captures avant et après traitement dans les parcelles traitées est 
Tableau III. Effectifs, évolution pré/post traitement des captures et analyses statistiques sur les Erigonidae en potspièges.

\begin{tabular}{|c|c|c|c|c|c|c|c|c|c|c|c|c|}
\hline & \multicolumn{3}{|c|}{$\begin{array}{c}\text { Témoin } \\
\text { Sous-parcelles }\end{array}$} & \multicolumn{3}{|c|}{$\begin{array}{l}\text { Deltaméthrine } \\
\text { Sous-parcelles }\end{array}$} & \multicolumn{3}{|c|}{$\begin{array}{c}\text { Phosalone } \\
\text { Sous-parcelles }\end{array}$} & \multicolumn{3}{|c|}{$\begin{array}{c}\text { Tests } \\
\text { entre les parcelles }\end{array}$} \\
\hline & 1 & 2 & 3 & 1 & 2 & 3 & 1 & 2 & 3 & $D T$ & $P T$ & $D P$ \\
\hline \multicolumn{13}{|l|}{1983} \\
\hline Période 1 & 11 & 11 & 6 & 10 & 8 & 2 & 6 & 4 & 4 & NS & NS & NS \\
\hline Période 2 & 12 & 7 & 6 & 28 & 13 & 13 & 14 & 10 & 9 & NS & NS & NS \\
\hline \multicolumn{13}{|l|}{ Traitement } \\
\hline Période 3 & 18 & 38 & 20 & 9 & 4 & 3 & 33 & 28 & 17 & ++ & NS & \pm \\
\hline Période 4 & 41 & 25 & 28 & 17 & 7 & 12 & 29 & 37 & 30 & ++ & NS & \pm \\
\hline Période 5 & 54 & 36 & 28 & 23 & 14 & 26 & 34 & 31 & 40 & NS & NS & NS \\
\hline$\%$ ap/av TT & \multicolumn{3}{|c|}{$+443,4 \%$} & \multicolumn{3}{|c|}{$+55,4 \%$} & \multicolumn{3}{|c|}{$+493,6 \%$} & & & \\
\hline 1985 & & & & & & & \multicolumn{3}{|c|}{ Diméthoate } & $D T$ & $R T$ & $D R$ \\
\hline Période 1 & 3 & 4 & 1 & 7 & 7 & 2 & 4 & 6 & 4 & NS & NS & NS \\
\hline Période 2 & 12 & 2 & 2 & 14 & 5 & 12 & 9 & 9 & 11 & NS & NS & NS \\
\hline \multicolumn{13}{|l|}{ Traitement } \\
\hline Période 3 & 20 & 8 & 12 & 3 & 2 & 6 & 11 & 4 & 5 & NS & NS & NS \\
\hline Période 4 & 25 & 15 & 29 & 1 & 7 & 5 & 11 & 11 & 18 & ++ & NS & \# \\
\hline Période 5 & 28 & 22 & 23 & 12 & 8 & 8 & 36 & 21 & 22 & ++ & NS & \pm \\
\hline Période 6 & 29 & 27 & 23 & 27 & 13 & 10 & 68 & 30 & 60 & ++ & $*$ & \pm \\
\hline$\%$ ap/av TT & \multicolumn{3}{|c|}{$+987,5 \%$} & \multicolumn{3}{|c|}{$+117 \%$} & \multicolumn{3}{|c|}{$+590,7 \%$} & & & \\
\hline
\end{tabular}

Signification des abréviations : voir tableau II.

nettement inférieure à celle enregistrée simultanément dans le témoin et va dans le même sens que les résultats de l'analyse statistique.

Oedothorax apicatus (tableau V) est également une araignée agrophile, très abondante en céréaliculture dans le Bassin parisien (Cocquempot et Chambon, 1989a). Pour cette espèce, un effet temporaire de la deltaméthrine apparaît en 1983 durant la période 3 . II traduit probablement une simple baisse d'activité car l'évolution des captures après traitement reste comparable, tout en étant plus faible, à celle notée dans le témoin (+ $450 \%$ contre $+547 \%$ ).

En 1985, l'hétérogénéité mise en évidence avant le traitement nous conduit à considérer les résultats avec une certaine réserve. Cette annéelà les populations d'O apicatus des parcelles deltaméthrine ont vu leurs effectifs significativement réduits au cours des périodes 4,5 et 6 . Le diméthoate ne paraît pas avoir d'effet, seul un excédent apparaît en fin d'étude par rapport au témoin, rappelant ainsi l'hétérogénéité observée en deuxième période.

Les pourcentages de captures après et avant traitement montrent également qu'il existe une réelle différence dans l'évolution des captures des 3 parcelles. Ils sont nettement plus faibles dans la deltaméthrine, confirmant ainsi le résultat de l'analyse statistique. La valeur significativement plus élevée en sixième période dans le diméthoate ne semble pas avoir d'incidence au niveau des pourcentages qui restent nettement plus faibles que ceux du témoin.

L'interprétation de ces résultats semble la même que celle émise pour l'ensemble des Erigonidae. Les effectifs significativement supérieurs enregistrés avant traitement dans la parcelle diméthoate se retrouvent en période 6 . Ceci permet de penser à l'existence d'une action réductrice du diméthoate sur l'activité d' $O$ apicatus durant les 3 premières périodes après traitement.

\section{Les Theridiidae (Theridion bimaculatum) (tableau VI)}

Theridion bimaculatum est la seule espèce, évoluant au niveau supérieur de la végétation, capturée en nombre suffisant pour permettre une analyse et illustrer l'impact des insecticides sur les Theridiidae. 
Tableau IV. Effectifs, évolution pré/post traitement des captures et analyses statistiques sur Erigone atra en potspièges.

\begin{tabular}{|c|c|c|c|c|c|c|c|c|c|c|c|c|}
\hline & \multicolumn{3}{|c|}{$\begin{array}{c}\text { Témoin } \\
\text { Sous-parcelles }\end{array}$} & \multicolumn{3}{|c|}{$\begin{array}{l}\text { Deltaméthrine } \\
\text { Sous-parcelles }\end{array}$} & \multicolumn{3}{|c|}{$\begin{array}{c}\text { Phosalone } \\
\text { Sous-parcelles }\end{array}$} & \multicolumn{3}{|c|}{$\begin{array}{c}\text { Tests } \\
\text { entre les parcelles }\end{array}$} \\
\hline & 1 & 2 & 3 & 1 & 2 & 3 & 1 & 2 & 3 & $D T$ & $P T$ & $D P$ \\
\hline \multicolumn{13}{|l|}{1983} \\
\hline Période 1 & 5 & 5 & 4 & 7 & 3 & 0 & 0 & 4 & 1 & NS & NS & NS \\
\hline Période 2 & 4 & 5 & 1 & 11 & 5 & 3 & 8 & 5 & 4 & NS & NS & NS \\
\hline \multicolumn{13}{|l|}{ Traitement } \\
\hline Période 3 & 10 & 22 & 10 & 6 & 1 & 1 & 18 & 17 & 14 & ++ & NS & $\#$ \\
\hline Période 4 & 23 & 13 & 17 & 5 & 4 & 8 & 19 & 20 & 15 & ++ & NS & $\#$ \\
\hline Période 5 & & 15 & 14 & 8 & 6 & 7 & 11 & 11 & 14 & ++ & ++ & \pm \\
\hline$\%$ ap/av $\pi$ & \multicolumn{3}{|c|}{$+504,2 \%$} & \multicolumn{3}{|c|}{$+48,3 \%$} & \multicolumn{3}{|c|}{$+531,8 \%$} & & & \\
\hline 1985 & & & & & & & \multicolumn{3}{|c|}{ Diméthoate } & $D T$ & $R T$ & $D R$ \\
\hline Période 1 & 1 & 0 & 0 & 4 & 4 & 2 & 1 & 2 & 2 & $*$ & $* *$ & \pm \\
\hline Période 2 & 6 & 0 & 0 & 7 & 3 & 6 & 4 & 3 & 3 & NS & NS & NS \\
\hline \multicolumn{13}{|l|}{ Traitement } \\
\hline Période 3 & 2 & 4 & 3 & 1 & 2 & 1 & 0 & 0 & 1 & ++ & ++ & $\infty$ \\
\hline Période 4 & 15 & 5 & 10 & 0 & 0 & 0 & 2 & 1 & 5 & ++ & ++ & $\#$ \\
\hline Période 5 & 7 & 5 & 3 & 5 & 0 & 3 & 3 & 4 & 1 & NS & NS & NS \\
\hline Période 6 & 6 & 8 & 3 & 7 & 6 & 3 & 3 & 4 & 4 & NS & NS & NS \\
\hline$\%$ ap/av TT & \multicolumn{3}{|c|}{$+914,3 \%$} & \multicolumn{3}{|c|}{$+7,7 \%$} & \multicolumn{3}{|c|}{$+86,7 \%$} & & & \\
\hline
\end{tabular}

Signification des abréviations : voir tableau II.

Les chiffres obtenus par les prélèvements au D-VAC de 1985 montrent l'existence d'un effet réducteur très fort des 2 matières actives. Les différences significatives enregistrées ne se manisfestent que durant la période suivant le traitement. On note également des différences très significatives entre les 2 parcelles traitées montrant ainsi que la deltaméthrine réduit davantage le nombre des captures de $T$ bimaculatum dont une majorité de mâles qui sont plus mobiles à cette époque de l'année. L'étude des pourcentages de l'évolution des captures après et avant traitement corrobore les résultats obtenus par l'analyse statistique.

\section{Les Linyphiidae (Lepthyphantes tenuis (tableau VII) et Bathyphantes gracilis)}

Les Linyphiidae sont en nombre relativement élevé, mais les captures assez régulièrement réparties dans le temps n'atteignent que rarement à chaque période des niveaux suffisants pour être analysables. Un effet négatif de la deltaméthrine apparaît toutefois en 1985 pour la première période après traitement, mais celui-ci porte sur de faibles effectifs de captures en pots pièges et disparaît très vite. L'espèce principale, Lepthyphantes tenuis (tableau VII) dont le nombre de captures avant traitement était plus élevé dans les parcelles traitées, présente la même chute d'effectifs que l'ensemble de la famille, mais montre un surplus significatif de captures par rapport au témoin en période 4 . Ce phénomène peut s'expliquer par une inaction temporaire liée au traitement entraînant une chute des captures (aucune capture n'a été enregistrée au cours de la première période après traitement dans cette parcelle), suivie d'une reprise d'activité matérialisée par une forte augmentation des captures légèrement supérieure à celle notée dans les autres parcelles.

Nous avons également remarqué une augmentation significative des captures par rapport au témoin, dans la parcelle traitée au diméthoate, durant la troisième période. Cette faible augmentation peut s'expliquer par une éventuelle action excitatrice de la matière active sur des effectifs initiaux déjà légèrement supérieurs à ceux du témoin. 
Tableau V. Effectifs, évolution pré/post traitement des captures et analyses statistiques sur Oedothorax apicatus en pots-pièges.

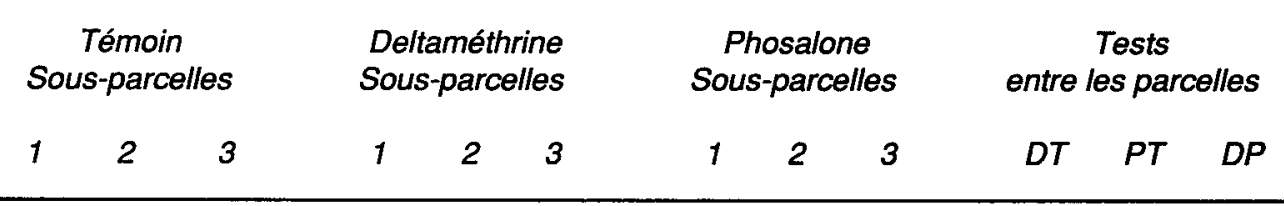

1983

\begin{tabular}{|c|c|c|c|c|c|c|c|c|c|c|c|c|}
\hline $\begin{array}{l}\text { Période } 1 \\
\text { Période } 2 \\
\text { Traitement }\end{array}$ & $\begin{array}{l}5 \\
5\end{array}$ & $\begin{array}{l}2 \\
2\end{array}$ & $\begin{array}{l}0 \\
1\end{array}$ & $\begin{array}{l}0 \\
3\end{array}$ & $\begin{array}{l}0 \\
4\end{array}$ & $\begin{array}{l}0 \\
3\end{array}$ & $\begin{array}{l}3 \\
2\end{array}$ & $\begin{array}{l}0 \\
4\end{array}$ & $\begin{array}{l}3 \\
2\end{array}$ & $\begin{array}{l}\text { NS } \\
\text { NS }\end{array}$ & $\begin{array}{l}\text { NS } \\
\text { NS }\end{array}$ & $\begin{array}{l}\text { NS } \\
\text { NS }\end{array}$ \\
\hline $\begin{array}{l}\text { Période } 3 \\
\text { Période } 4 \\
\text { Période } 5 \\
\% \text { ap/av TT }\end{array}$ & $\begin{array}{r}5 \\
17 \\
26 \\
+\end{array}$ & $\begin{array}{r}9 \\
6 \\
13 \\
46,7\end{array}$ & $\begin{array}{r}5 \\
5 \\
11\end{array}$ & $\begin{array}{r}3 \\
10 \\
12\end{array}$ & $\begin{array}{r}0 \\
3 \\
7 \\
45\end{array}$ & $\begin{array}{r}1 \\
4 \\
15\end{array}$ & $\begin{array}{r}5 \\
7 \\
19 \\
+\end{array}$ & $\begin{array}{r}5 \\
8 \\
17 \\
85,7\end{array}$ & $\begin{array}{r}2 \\
8 \\
25\end{array}$ & $\begin{array}{l}++ \\
\text { NS } \\
\text { NS }\end{array}$ & $\begin{array}{l}\text { NS } \\
\text { NS } \\
\text { NS }\end{array}$ & $\begin{array}{l} \pm \\
\text { NS } \\
\text { NS }\end{array}$ \\
\hline 1985 & & & & & & & \multicolumn{3}{|c|}{ Diméthoate } & $D T$ & $R T$ & $D R$ \\
\hline $\begin{array}{l}\text { Période } 1 \\
\text { Période } 2\end{array}$ & $\begin{array}{l}1 \\
2\end{array}$ & $\begin{array}{l}1 \\
1\end{array}$ & $\begin{array}{l}0 \\
1\end{array}$ & $\begin{array}{l}1 \\
1\end{array}$ & $\begin{array}{l}2 \\
1\end{array}$ & $\begin{array}{l}0 \\
0\end{array}$ & $\begin{array}{l}2 \\
3\end{array}$ & $\begin{array}{l}1 \\
2\end{array}$ & $\begin{array}{l}1 \\
4\end{array}$ & $\begin{array}{l}\text { NS } \\
+\end{array}$ & NS & $\begin{array}{l}\text { NS } \\
\pm\end{array}$ \\
\hline \multicolumn{13}{|l|}{ Traitement } \\
\hline $\begin{array}{l}\text { Période } 3 \\
\text { Période } 4 \\
\text { Période } 5 \\
\text { Période } 6\end{array}$ & $\begin{array}{r}14 \\
6 \\
17 \\
19\end{array}$ & $\begin{array}{r}3 \\
6 \\
16 \\
15\end{array}$ & $\begin{array}{r}7 \\
13 \\
17 \\
19\end{array}$ & $\begin{array}{r}1 \\
0 \\
5 \\
14\end{array}$ & $\begin{array}{l}0 \\
3 \\
5 \\
7\end{array}$ & $\begin{array}{l}2 \\
3 \\
4 \\
7\end{array}$ & $\begin{array}{r}7 \\
7 \\
27 \\
58\end{array}$ & $\begin{array}{r}2 \\
6 \\
15 \\
18\end{array}$ & $\begin{array}{r}3 \\
11 \\
17 \\
42\end{array}$ & $\begin{array}{l}\text { NS } \\
++ \\
++ \\
+\end{array}$ & $\begin{array}{l}\text { NS } \\
\text { NS } \\
\text { NS } \\
*\end{array}$ & $\begin{array}{l}\text { NS } \\
\# \\
\# \\
\#\end{array}$ \\
\hline$\%$ ap/av TT & \multicolumn{3}{|c|}{$+2433,3 \%$} & \multicolumn{3}{|c|}{$+920 \%$} & \multicolumn{3}{|c|}{$+1538,5 \%$} & & & \\
\hline
\end{tabular}

Signification des abréviations : voir tableau II.

Bathyphantes gracilis est significativement affecté par la deltaméthrine au cours de la quatrième période de 1983, mais les effectifs très faibles ne permettent pas d'interpréter ces résultats.

\section{Autres familles et espèces}

Les effectifs de captures des nombreuses autres espèces, même regroupés au niveau de la famille, ne permettent pas d'effectuer les

Tableau VI. Effectifs, évolution pré/post traitement des captures et analyses statistiques sur Theridion bimaculatum au D-VAC.

\begin{tabular}{|c|c|c|c|c|c|c|c|c|c|c|c|c|}
\hline \multirow[t]{2}{*}{$\begin{array}{c}1985 \\
D-V A C\end{array}$} & \multicolumn{3}{|c|}{$\begin{array}{c}\text { Témoin } \\
\text { Sous-parcelles }\end{array}$} & \multicolumn{3}{|c|}{$\begin{array}{l}\text { Deltaméthrine } \\
\text { Sous-parcelles }\end{array}$} & \multicolumn{3}{|c|}{$\begin{array}{c}\text { Diméthoate } \\
\text { Sous-parcelles }\end{array}$} & \multicolumn{3}{|c|}{$\begin{array}{c}\text { Tests } \\
\text { entre les parcelles }\end{array}$} \\
\hline & 1 & 2 & 3 & 1 & 2 & 3 & 1 & 2 & 3 & $D T$ & $P T$ & $D R$ \\
\hline Période 2 & 5 & 9 & 13 & 11 & 6 & 7 & 9 & 14 & 22 & NS & NS & NS \\
\hline \multicolumn{13}{|l|}{ Traitement } \\
\hline $\begin{array}{l}\text { Période } 3 \\
\text { Période } 4 \\
\text { Période } 5 \\
\text { Période } 6\end{array}$ & $\begin{array}{r}30 \\
5 \\
10 \\
1\end{array}$ & $\begin{array}{r}33 \\
3 \\
9 \\
0\end{array}$ & $\begin{array}{r}18 \\
5 \\
10 \\
0\end{array}$ & $\begin{array}{l}5 \\
4 \\
9 \\
1\end{array}$ & $\begin{array}{l}4 \\
1 \\
3 \\
0\end{array}$ & $\begin{array}{l}4 \\
2 \\
3 \\
0\end{array}$ & $\begin{array}{r}17 \\
2 \\
6 \\
0\end{array}$ & $\begin{array}{r}14 \\
1 \\
13 \\
0\end{array}$ & $\begin{array}{r}16 \\
4 \\
6 \\
2\end{array}$ & $\begin{array}{l}++ \\
\text { NS } \\
\text { NS } \\
\text { NS }\end{array}$ & $\begin{array}{l}++ \\
\text { NS } \\
\text { NS } \\
\text { NS }\end{array}$ & $\begin{array}{l}\# \\
\text { NS } \\
\text { NS } \\
\text { NS }\end{array}$ \\
\hline$\%$ ap/av TT & \multicolumn{3}{|c|}{$+359,3 \%$} & \multicolumn{3}{|c|}{$+50 \%$} & \multicolumn{3}{|c|}{$+80 \%$} & & & \\
\hline
\end{tabular}

Signification des abréviations : voir tableau II. 
Tableau VII. Effectifs, évolution pré/post traitement des captures et analyses statistiques sur Lethyphantes tenuis en pots-pièges.

\begin{tabular}{|c|c|c|c|c|c|c|c|c|c|c|c|c|}
\hline & \multicolumn{3}{|c|}{$\begin{array}{c}\text { Témoin } \\
\text { Sous-parcelles }\end{array}$} & \multicolumn{3}{|c|}{$\begin{array}{l}\text { Deltaméthrine } \\
\text { Sous-parcelles }\end{array}$} & \multicolumn{3}{|c|}{$\begin{array}{c}\text { Diméthoate } \\
\text { Sous-parcelles }\end{array}$} & \multicolumn{3}{|c|}{$\begin{array}{c}\text { Tests } \\
\text { entre les parcelles }\end{array}$} \\
\hline & 1 & 2 & 3 & 1 & 2 & 3 & 1 & 2 & 3 & $D T$ & $P T$ & $D R$ \\
\hline \multicolumn{13}{|l|}{1985} \\
\hline $\begin{array}{l}\text { Période } 1 \\
\text { Période } 2\end{array}$ & $\begin{array}{l}8 \\
3\end{array}$ & $\begin{array}{l}1 \\
1\end{array}$ & $\begin{array}{l}0 \\
0\end{array}$ & $\begin{array}{l}2 \\
3\end{array}$ & $\begin{array}{l}3 \\
3\end{array}$ & $\begin{array}{l}2 \\
7\end{array}$ & $\begin{array}{l}1 \\
5\end{array}$ & $\begin{array}{l}5 \\
5\end{array}$ & $\begin{array}{l}0 \\
0\end{array}$ & $\begin{array}{l}\text { NS } \\
\text { NS }\end{array}$ & $\begin{array}{l}\text { NS } \\
\text { NS }\end{array}$ & $\begin{array}{l}\text { NS } \\
\text { NS }\end{array}$ \\
\hline \multicolumn{13}{|l|}{ Traitement } \\
\hline $\begin{array}{l}\text { Période } 3 \\
\text { Période } 4 \\
\text { Période } 5 \\
\text { Période } 6\end{array}$ & $\begin{array}{r}2 \\
1 \\
8 \\
10\end{array}$ & $\begin{array}{l}1 \\
1 \\
1 \\
1\end{array}$ & $\begin{array}{l}1 \\
3 \\
3 \\
1\end{array}$ & $\begin{array}{l}0 \\
4 \\
8 \\
6\end{array}$ & $\begin{array}{r}0 \\
5 \\
5 \\
10\end{array}$ & $\begin{array}{r}0 \\
7 \\
10 \\
5\end{array}$ & $\begin{array}{l}1 \\
4 \\
3 \\
8\end{array}$ & $\begin{array}{l}4 \\
2 \\
3 \\
3\end{array}$ & $\begin{array}{l}4 \\
2 \\
2 \\
8\end{array}$ & $\begin{array}{l}++ \\
\star \star \\
\text { NS } \\
\text { NS }\end{array}$ & $\begin{array}{l}\star \\
\text { NS } \\
\text { NS } \\
\text { NS }\end{array}$ & $\begin{array}{l}+\# \\
\infty \\
\text { NS } \\
\text { NS }\end{array}$ \\
\hline$\%$ ap/av $\pi$ & \multicolumn{3}{|c|}{$+153,8 \%$} & \multicolumn{3}{|c|}{$+200 \%$} & \multicolumn{3}{|c|}{$+175 \%$} & & & \\
\hline
\end{tabular}

Signification des abréviations : voir tableau II.

tests statistiques ou ne sont pas suffisants pour que l'on puisse apprécier la valeur réelle des résultats. Quelques remarques peuvent néanmoins être faites.

- aucune action n'a été remarquée sur les Tetragnathidae;

- nous avons noté quelques effets sur les effectifs d'immatures capturés au D-VAC. La diversité des espèces regroupées sous cette appellation ainsi que leur comportement différent de celui des adultes nous commandent d'être prudents dans l'interprétation de ces écarts qui sont limités dans le temps et dans l'espace.

\section{CONCLUSION - DISCUSSION}

À l'issue de ces 3 années d'observations, il apparaît que les traitements insecticides mettant en œuvre la deltaméthrine, la phosalone et le diméthoate ont une incidence mesurable sur la faune aranéologique des cultures de blé.

Précisons que les expériences conduites à Saint-Cyr-en-Arthies ne mesurent pas la toxicité directe des matières actives sur les araignées mais enregistrent des variations de niveaux de captures qui n'impliquent pas nécessairement la mort des individus lors de la chute des effectifs mais peuvent être le simple reflet d'une variation de leur niveau d'activité.

De cette étude, il ressort que sur les 110 espèces recensées, seules quelques-unes présen- tent des effectifs suffisants pour réaliser une analyse statistique des données et, hormis Theridion bimaculatum, vivent dans les strates basses ou sur le sol.

Nous avons constaté des chutes de captures significatives dans les parcelles traitées à la deltaméthrine pour Pardosa prativaga, les Erigonidae en général, Erigone atra, Oedothorax apicatus, Theridion bimaculatum, Lepthyphantes tenuis et Bathyphantes gracilis. Cette action touche pratiquement toutes les araignées les plus abondamment appréhendées au cours de l'expérimentation, mais varie sensiblement en fonction des espèces et des années. La durée d'action est également très variable allant d' 1 à 3 périodes après traitement ( 1 à 3 semaines environ). À l'exception de Pardosa prativaga en 1984, l'effet de la deltaméthrine se traduit par une différence importante du niveau de progression des captures après traitement par rapport au témoin. II apparaît donc que cette matière active pertube les araignées suffisamment pour modifier leur comportement vis-à-vis des techniques de captures jusqu'à l'arrêt des prélèvements sans que l'on sache l'exact devenir de ces populations et si cette baisse du nombre des captures est liée à une mortalité, une fuite ou une activité contrariée des différentes araignées.

Les 2 années d'utilisation de la phosalone ont permis de noter que cette matière active a eu un effet limité d'une semaine sur Pardosa prativaga et Erigone atra en 1983. Dans ces 2 cas, cet 
effet n'occasionne qu'une retenue passagère de l'activité des araignées, celles-ci retrouvant une progression des niveaux de captures après traitement comparable à l'activité du témoin. II est donc fort probable qu'il n'y a pas de mortalité due à la phosalone mais une simple perturbation de l'activité matérialisée par une chute du nombre des captures dans la semaine suivant le traitement.

Les captures d'Erigone atra et de Theridion bimaculatum ont été significativement affectées par le diméthoate en 1985. Le pourcentage des captures après et avant traitement montre que les parcelles traitées avec cette matière active n'ont pas une progression du niveau de captures équivalente à celle du témoin. II y a donc un décalage durable dans la dynamique des captures après traitement par rapport au témoin qui correspond à une modification de l'activité liée aux mêmes hypothèses que celles émises pour la deltaméthrine.

Les exemples d'effets des traitements insecticides sont plus abondants pour la deltaméthrine que pour la phosalone ou le diméthoate mais ils portent sur 3 années expérimentales contre 2 à la phosalone et 1 seule au diméthoate. II convient donc d'être prudent dans l'interprétation de la comparaison des résultats entre les différentes matières actives.

Ces résultats concordent avec ceux de Powell et al et de De Clercq et Pietraszko (1983) qui ont observé d'une part, un effet limité du diméthoate à la première semaine après traitement et d'autre part, une action en petite parcelle fermée limitée à un maximum de $11 \%$ de réduction des captures d'araignées sur l'ensemble de la période post-traitement.

Vickerman et Sunderland (1977) ont noté une réduction de $90 \%$ du nombre d'araignées une semaine après traitement au diméthoate, suivie d'une limitation à $50 \%$ jusqu'à la fin des observations. Cette étude a été réalisée à l'aide du $D$ VAC et ses résultats sont comparables à ceux que nous avons obtenus pour Theridion bimaculatum en 1985, soit une action immédiate très forte qui ne permet pas aux populations aranéologiques des parcelles traitées de retrouver un niveau similaire à celles du témoin. On notera toutefois que l'action immédiate du traitement au diméthoate est de $90 \%$ pour nos collègues contre $50 \%$ environ dans notre étude. II est par contre très intéressant de noter que ces mêmes auteurs ont constaté une mortalité directe notamment pour Erigone atra, Bathyphantes gracilis, Lepthyphantes tenuis, des espèces du genre Oedothorax Bertkau, Theridion bimaculatum et 2 es- pèces de Pardosa CL Koch, ce qui peut expliquer en partie la faiblesse des niveaux de progression de captures post-traitement de certaines espèces dans notre étude.

Le travail de Basedow et al (1985) met en évidence une action de la deltaméthrine plus marquée que celle notée dans notre étude. II est probable que l'utilisation de cette matière active à $7,5 \mathrm{~g} \mathrm{ma} / \mathrm{ha}$ au lieu de $6,5 \mathrm{~g} \mathrm{ma} / \mathrm{ha}$ soit en grande partie responsable du plus grand effet défavorable observé par ces auteurs sur les Linyphiidae sensu lato (= Erigonidae + Linyphiidae). II est par contre difficile de justifier les différences de captures par l'unique biais de la mortalité des araignées ainsi que l'augmentation très forte en fin d'étude par l'unique fait d'une recolonisation et, sans ignorer ces éventualités, nous pensons qu'une modification de l'activité a également une bonne part de responsabilité dans la chute ou l'augmentation de niveaux de captures des araignées.

Au terme de cette étude, il apparaît nettement que, pour les espèces d'araignées abondamment capturées, les matières actives testées ont une incidence variable selon les années et souvent de courte durée.

La baisse des niveaux de captures peut traduire, soit la mortalité des araignées, qu'on ne peut appréhender par la méthode utilisée, soit une fuite vers d'autre biotopes avoisinants, soit une variation du niveau d'activité. Cette dernière se manifesterait d'abord par un arrêt ou ralentissement temporaire suivi dans bien des cas d'une reprise d'activité comparable à celle du témoin, 1 à 2 périodes après le traitement. II n'en résulte pas moins, que les araignées soient tuées ou simplement inactivées, l'action prédatrice sur les pucerons ou autres phytophages, se voit freinée, voire annulée à court terme par les traitements. Les conséquences de cette inactivité seront négligeables, surtout si l'on considère que les matière actives utilisées ont fortement réduit les populations de pucerons.

La question se pose de savoir comment, en dehors de cet aspect prédation, la perturbation amenée dans le comportement des araignées peut avoir des conséquences au niveau de la reproduction et de la dynamique de population, car les traitements interviennent à une période d'intense activité sexuelle, pour beaucoup des espèces recensées. Une réduction directe ou indirecte du nombre des araignées d'une biocénose céréalière peut contribuer à réduire l'action précoce de ces prédateurs sur les populations de ravageurs de l'année suivante par 
manque d'effectifs printaniers et par conséquence favoriser, d'année en année, la colonisation et la dynamique des populations des insectes nuisibles.

La réponse à ces questions nécessitera le développement d'études bio-écologiques sur les araignées pour lesquelles, dans de nombreux cas, nous avons très peu, voire pas d'informations.

\section{RÉFÉRENCES}

Basedow T, Rzehak H, Voss K (1985) Studies on the effect of deltamethrin sprays on the numbers of epigeal predatory arthropods occuring in arable fields. Pestic Sci 16, 325-331

Cocquempot C, Chambon JP (1989a) Inventaire aranéologique des biocénoses céréalières du Bassin parisien. Boll Zool Agrir Bachic Ser II, 21, 27-43

Cocquempot C, Chambon JP (1989b) Importance des araignées parmi les prédateurs polyphages des biocénoses céréalières du Bassin parisien. Def Veg 257, 13-16

De Clercq R, Pietraszko R (1983) On the influence of pesticides on the epigeal arthropod fauna in winter wheat. Proc Int Conf Intergr Plant Prot 1, 41-44

Fischer L (1987) Étude comparée de l'incidence de trois insecticides deltaméthrine, diméthoate, phosalone sur la faune des Arthropodes épigés des agrosystèmes céréaliers : Cas d'un traitement à la floraison en culture de blé d'hiver. Thèse $3 e$ cycle, Univ Paris XI, $325 \mathrm{p}+$ annexes

Fischer L, Chambon JP (1987a) Faunistical inventory of cereal Arthropods after flowering and incidence of insecticide treatments with deltamethrin, dime- thoate and phosalone on epigeal fauna. Meded Fac Landbouwwet Rijksuniv Gent 52, 201-211

Fischer L, Chambon JP (1987b) Effect of an insecticide treatment at flowering stage on the epigeic fauna of a wheat culture. CR Conf Int Ravageurs Agric, Paris, ANPP 6 (II/III), 137-148

Ledoux JC, Canard A (1981) Initiation à l'étude systématique des Araignées. (Ledoux ed) Dommazan, $56 \mathrm{p}$

Locket GH, Millidge AF (1951-1953) British Spiders. Ray Soc London 1951 I, 310 p; 1953 II, 449p

Locket GH, Millidge AF, Merret P (1974) British Spiders. Ray Soc London 1974 III, 314p

Powell W, Dean GJ, Dewar A (1985) Effects of pirimicarb, dimethoate and benomyl on natural enemies of cereal aphids in winter wheat. $J$ Appl Biol 106, 235-242

Reynaud P (1989) Étude comparée de l'incidence de trois insecticides sur la faune des Araneae des agrosystèmes céréaliers. DAA, ENSSAA Dijon, 54 $\mathrm{p}+$ annexes

Simon E (1914-1932) Les Arachnides de France VI, Roret Paris, $1298 \mathrm{p}$

Snedecor GW, Cochran WG (1957 Méthodes statistiques. ACTA, Paris, $649 \mathrm{p}$

Tongiorgi $P$ (1966) Italian Wolf Spiders of the genus Pardosa (Araneae, Lycosidae). Bull Mus Comp Zool 134, 275-334

Vickerman GP, Sunderland KD (1977) Some effects of dimethoate on Arthropods in winter wheat. $J$ Appl Ecol 14, 767-777

Wiehle H (1956) Linyphiidae (Baldachinspinnen). Die Tierwelt Deutschlands, Spinnentiere oder Arachnoidea (Araneae) X, $337 p$

Wiehle H (1960) Micryphantidae (Zwergspinnen). Die Tierwelt Deutschlands, Spinnentiere oder Arachnoidea (Araneae) XI, $620 p$ 